\title{
Incidence Patterns and Outcomes of Ewing Sarcoma in South Korea (1999-2017): A Retrospective Analysis Using Korea Central Cancer Registry Data
}

\author{
Jun Ah Lee', Jiwon Lim², Dayeon Park'², Hye Young Jin', Meerim Park¹, Hyeon Jin Park', Jong Woong Park ${ }^{3}$, June Hyuk Kim³, \\ Hyun Guy Kang ${ }^{3}$, Young-Joo Won ${ }^{2}$ \\ ${ }^{1}$ Department of Pediatrics, Center for Pediatric Center, National Cancer Center, Goyang, ${ }^{2}$ Division of Cancer Registration and Surveillance, National \\ Cancer Center, Goyang, ${ }^{3}$ Orthopaedic Oncology Clinic, Center for Rare Cancer, National Cancer Center, Goyang, Korea
}

Purpose Due to low incidence, epidemiologic data of Ewing sarcoma in the Asian population are scarce. We aimed to examine the incidence pattern and outcome of patients with Ewing sarcoma in the Republic of Korea.

Materials and Methods Data of patients with Ewing sarcoma diagnosed between 1999 and 2017 were obtained from the Korea Central Cancer Registry (KCCR). Incidence, clinical characteristics, and survival rates were analyzed and compared between different age groups.

Results There were 788 cases (459 males, 329 females), with a median age at diagnosis of 20 years. The age-standardized rate of Ewing sarcoma was 1.01. The number of cases and incidence rates in each age group were as follows: children, 1.6; adolescents and young adults (AYA), 0.93; adults, 0.44; and elderly, 0.53. There were more male cases in children and the AYA group ( $<<0.001)$. Extraskeletal tumors $(p<0.001)$, primary sites other than extremity $(p=0.007)$, and presence of metastasis at diagnosis $(p=0.031)$ were more frequent in the adults and elderly group. With a median survival time of 78 months, the 5-year overall survival (OS) rate of the entire cohort was 52\%. Children fared best (5-year OS, 75\%), and the 5-year OS of AYA patients (51\%) approximated the 0S of the entire cohort. A two-fold difference of 5-year OS was observed between adults and elderly patients (42\% vs. 19\%). On univariate and multivariate analyses, age $\geq 15$ years and presence of metastasis were adverse prognostic factors.

Conclusion This was the first epidemiologic study of Ewing sarcoma using the KCCR data. With a similar incidence to other Asian countries, the survival rate was slightly lower than that of Euro-American cases. Collaborative clinical studies are necessary to improve the outcome of Ewing sarcoma in low-incidence populations.

Key words Ewing sarcoma, Korea, Low-incidence, Epidemiology

\section{Introduction}

Ewing sarcoma is a rare cancer, usually occurring in children and adolescents [1]. The incidence of Ewing sarcoma differs among racial groups [1-3]. The United States data show a 9-fold difference in incidence between African Americans and Caucasians [2]. The incidence of Ewing sarcoma in China is 2 or 3 times lower than that in European and American countries [4-6]. Epidemiologic data on Ewing sarcoma in low-incidence populations are scarce. Moreover, current knowledge about Ewing sarcoma is mainly based on studies of case series and clinical trials involving children and adolescents [7-11]. Adults and elderly patients are usually treated with the same multidisciplinary approach as children. However, limited data exists on the incidence patterns and outcomes of Ewing sarcoma in older age groups.

The Korea Central Cancer Registry (KCCR) maintains and provides extensive population-based epidemiologic data. In this study, we aimed to examine the incidence pattern and outcome of patients with Ewing sarcoma diagnosed between 1999 and 2017. We sought to answer the following questions: (1) What are the incidence and outcomes of Korean patients with Ewing sarcoma? and (2) Do older patients have different disease patterns and outcomes from younger patients?

\section{Materials and Methods}

\section{Data sources}

The KCCR was initiated as a nationwide, hospital-based cancer registry in 1980 by the Ministry of Health and Welfare, and collects data pertaining to the entire population since 1999. The Korea National Cancer Incidence Database (KNCIDB) is composed of the KCCR data and includes infor-

Correspondence: Young-Joo Won

Division of Cancer Registration and Surveillance, National Cancer Center, 323 Ilsan-ro, Ilsandong-gu, Goyang 10408, Korea

Tel: 82-31-920-2015 Fax: 82-31-920-2179 E-mail: astra67@ncc.re.kr

Received March 9, 2021 Accepted July 19, 2021 Published Online July 20, 2021

*Jun Ah Lee and Jiwon Lim contributed equally to this work. 
mation on the demographics of cancer patients.

Ewing sarcoma was defined according to the International Classification of Disease for Oncology, 3rd edition. Data on the incidence and survival of Ewing sarcoma between 1999 and 2017 were obtained from the KNCIDB and Mortality data were derived from Statistics Korea [12]. Data on Ewing sarcoma patients, including age, sex, date of initial diagnosis, primary tumor site, tissue origin (skeletal or extraskeletal), stage at diagnosis, and initial treatment, were retrieved from the KCCR.

\section{Statistical analyses}

Patients were grouped by age: (1) children $\leq 14$ years, (2) adolescents and young adults (AYA) 15-39 years, (3) adults 40-64 years, and (4) elderly $\geq 65$ years. Age-specific incidence rates and age-standardized incidence rates (ASR) using Segi's world standard population were calculated and expressed per million [13]. Incidence rates and clinical characteristics were compared between different age groups based on sex, period of diagnosis (1999-2003, 2004-2008, 2009-2013, 2014-2017), site of origin (bone or soft tissue), tumor location, the Surveillance, Epidemiology, and End Results (SEER) summary stage [14] (since 2006), and the first course of treatment within 4 months after diagnosis.

Overall survival rates were calculated using the KaplanMeier method [15]. Survival differences among patients grouped according to clinical variables were compared using the log-rank test. The Cox proportional hazards model [16] was applied to estimate the effect of covariates on hazard rates. All statistical tests were two-tailed, and results were considered statistically significant at p-values $<0.05$. Statistical analyses were conducted using SAS software ver. 9.4 (SAS Institute Inc., Cary, NC) and STATA software ver. 16 (StataCorp LLC, College Station, TX).

\section{Results}

\section{Incidence and clinical characteristics}

The age-standardized incidence rate of Ewing sarcoma during the study period of 19 years was 1.01 (Table 1). There were 788 cases (459 men and 329 women), with a median age at diagnosis of 20 years. The incidence was highest in adolescents and young adults, specifically among teenagers and those in their twenties (Fig. 1). The number of cases and incidence rates in each age group were as follows: children, 260 cases, rate, 1.6; adolescents and young adults, 340 cases, rate, 0.93; adults, 138 cases, rate, 0.44; elderly, 50 cases, rate, 0.53 (Table 1). The number of newly diagnosed cases was similar between study periods; however, there were marginally more patients in the 2009-2013 period (Table 1). Ew- ing sarcoma originated from bones in 592 patients (75.1\%), and soft tissue in 196 patients $(24.9 \%)$. The primary tumor sites were the extremities in 282 patients $(35.8 \%)$, trunk in $363(46.1 \%)$, and elsewhere in $143(18.1 \%)$. Information about disease extent was included in the KCCR system from 2006, corresponding to 543 cases. Among them, disease extent was unknown in 119 cases, and 220 patients $(40.5 \%)$ had regional or distant metastasis at the time of diagnosis.

Treatment data were available for 499 patients, and treatment modalities were similar over the 19-year period. Local treatment corresponds to surgery and/or radiotherapy, and multidisciplinary treatment was defined as local treatment plus multi-agent systemic chemotherapy. Multidisciplinary treatment was administered in $68.8 \%$ of patients with extremity tumors, $72.9 \%$ with axial tumors, and $68.9 \%$ with tumors in other sites $(\mathrm{p}=0.609)$. However, local treatment modalities differed according to tumor sites. For patients with extremity tumors, $95.4 \%$ underwent surgery, and $4.6 \%$ received radiotherapy. Among cases with axial tumors, $86.4 \%$ received surgery and $13.6 \%$ radiotherapy. Similarly, $85.6 \%$ of tumor patients located elsewhere received surgery, and $14.4 \%$ radiotherapy $(\mathrm{p}=0.006)$.

Generally, older patients presented with clinical features associated with poor outcomes. There were more male cases and tumors originating in bones among children and AYA patients compared to adults and the elderly. The proportion of patients with tumors of extraskeletal origin, or tumors located in regions other than the extremities, and who had metastasis at diagnosis showed significant differences between the age groups (Table 1). Treatment information that was available for 499 cases, showed that multidisciplinary treatment was more frequently used in children and AYAs. Excluding unknown treatment, more adults $(n=32,36.8 \%)$ and the elderly $(n=18,60.0 \%)$ received local treatment only (surgery and/or radiotherapy). The proportion of patients who received multidisciplinary treatment was $63.2 \%(n=55)$ in adults and $40.0 \%(n=12)$ in the elderly. In contrast, 123 children $(75.0 \%)$ and 163 AYA patients $(74.8 \%)$ received local treatment as well as chemotherapy (Table 1).

\section{Survival}

Survival analysis was performed in 739 patients, excluding the 49 cases with missing data. The median survival time was 78 months, and the 5-year overall survival (OS) rate was $52 \%$. Survival was similar during the 19-year period, except in patients who were diagnosed in 1999 and 2002 (Fig. 2 ). There was no difference in the 5-year OS rates between patients with skeletal tumors and extra-skeletal tumors (52\% vs. 53\%). Survival was reciprocally correlated with age. The median survival times of AYA, adults, and elderly patients were 65,30 , and 9 months, respectively. Children fared the 


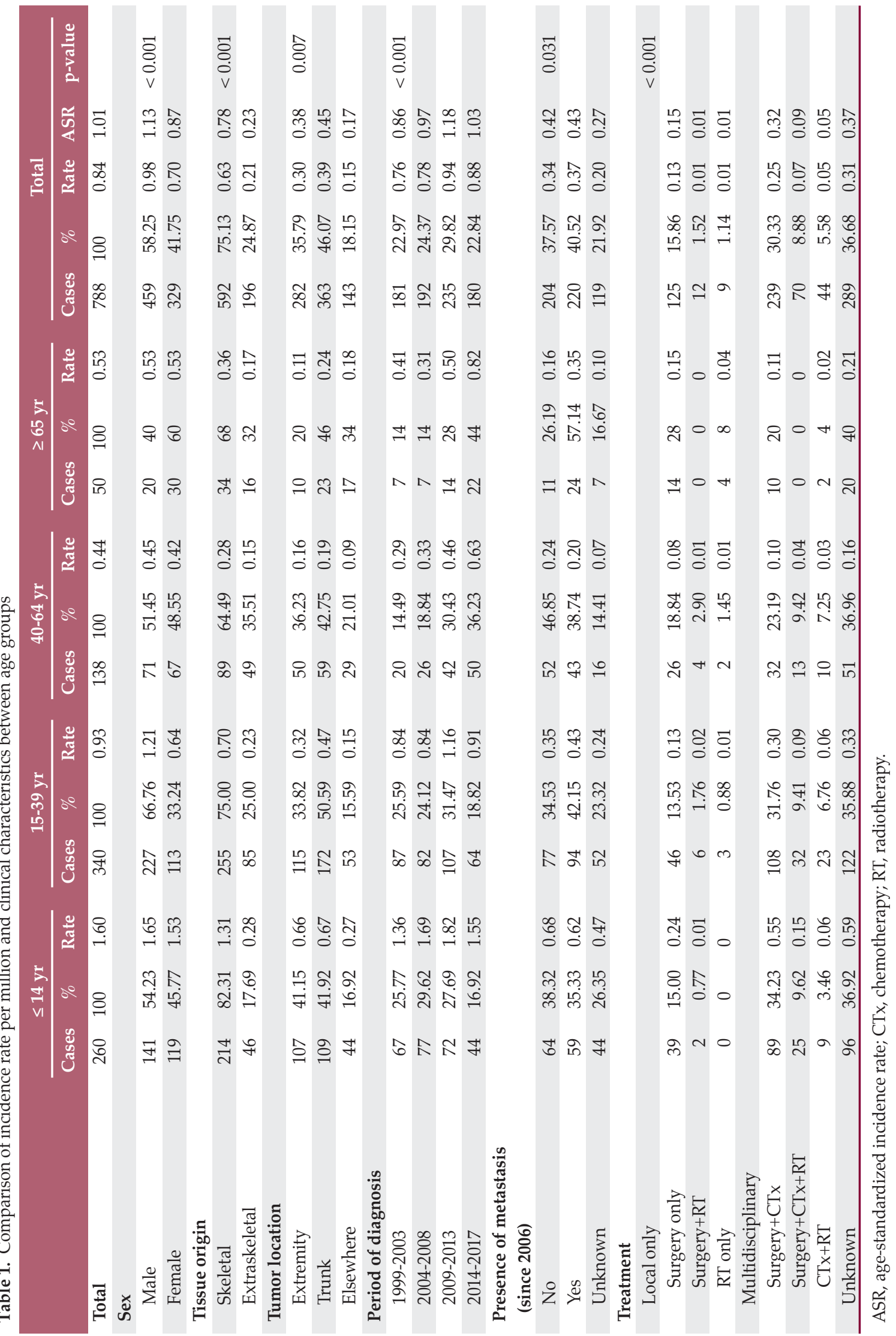




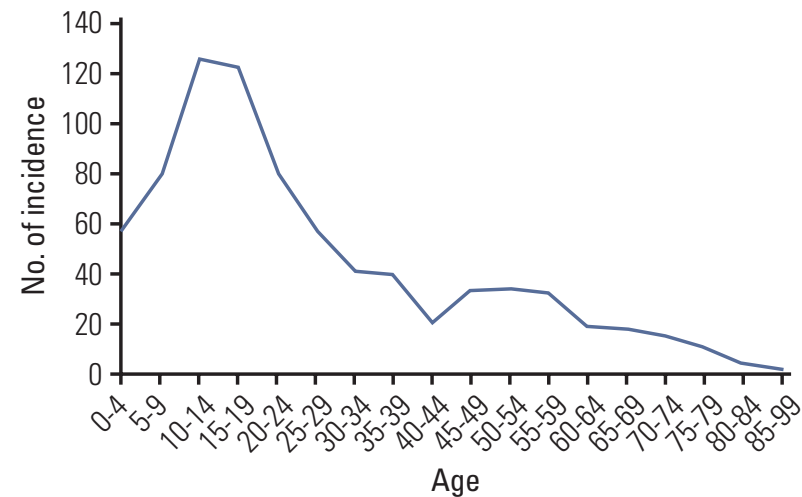

Fig. 1. Age distribution of Ewing sarcoma.
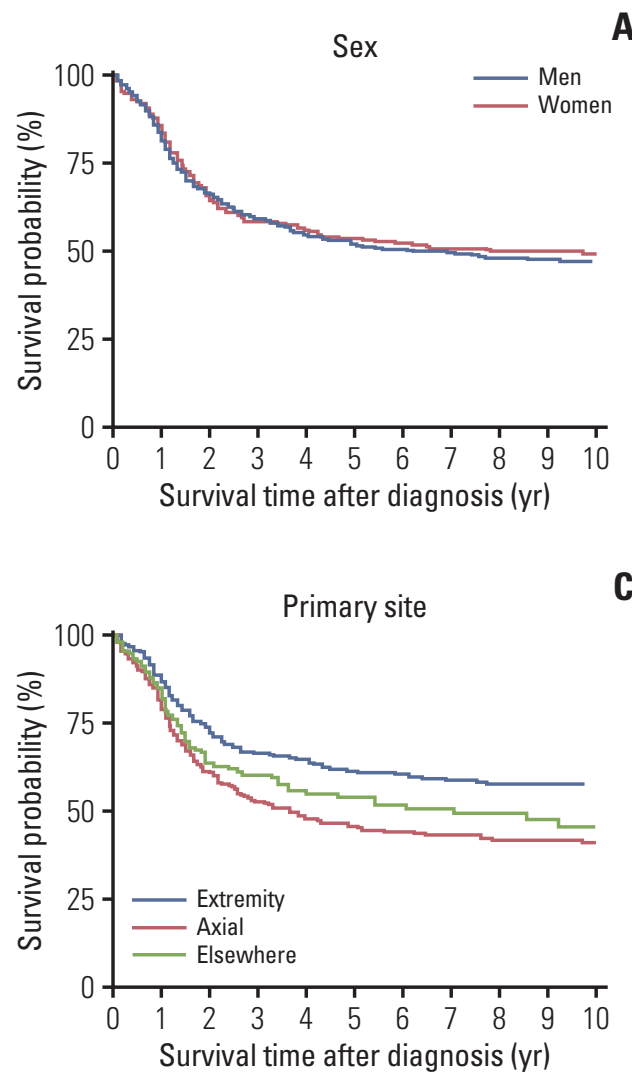

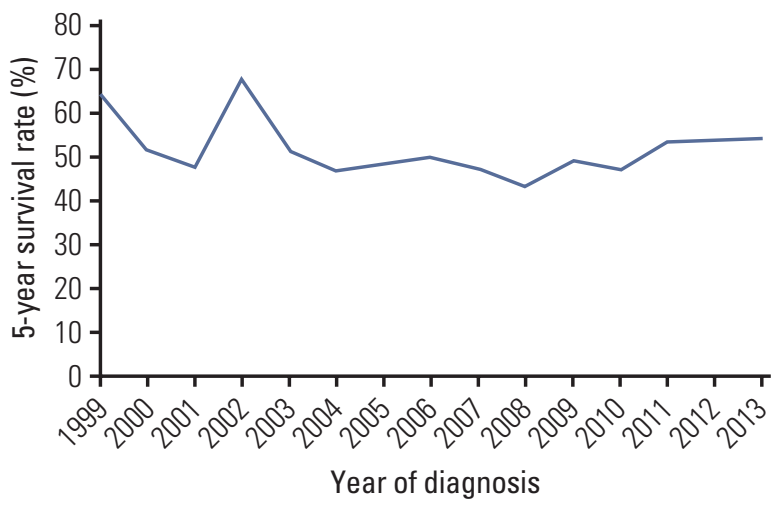

Fig. 2. The 5-year survival trend over the 19-year period.
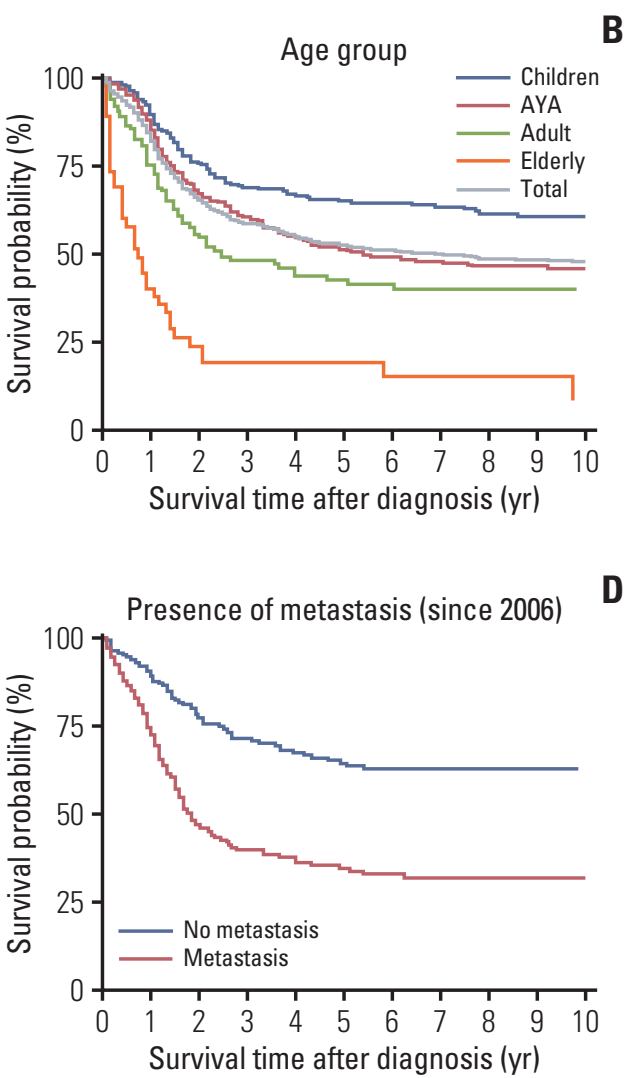

Fig. 3. (A-D) Comparison of overall survival. AYA, adolescents and young adults.

best (5-year OS, 64\%), and the 5-year OS of AYA patients was similar to that of the 739 cases reported during 19992017 (51\% vs. 52\%). A two-fold difference in survival was observed between adults and elderly patients (Fig. 3). In addition to age, the presence of metastasis and tumor location other than the extremities were adverse prognostic factors on univariate analysis. However, prognostic significance of tumor location was lost on multivariate analysis (Table
2). Unexpectedly, treatment modalities did not influence the survival of Ewing sarcoma patients (Table 2).

\section{Discussion}

Using data from the KCCR between 1999 and 2017, we analyzed the epidemiology of Ewing sarcoma in South 
Korea. While previous studies were based on small case series, our work represents the only population-based study on the incidence and outcome of Ewing sarcoma in South Korea. The incidence of Ewing sarcoma in South Korea was similar to that in other Asian countries, and the survival rate was slightly lower than that of Euro-American cases. Older age and presence of metastasis at diagnosis were associated with poor outcomes.

Due to the nature of KCCR, our study has several limitations. Information on disease extent (metastasis) has become available only since 2006. Detailed information on tumor (size), treatment, and co-morbidities of patients are not provided by KCCR data. Moreover, registered information on treatment was included only treatment during the first 4 months after diagnosis. Therefore, the prognostic significance of clinical variables should be considered to be limited within the context of the currently available data.

The ASR of Ewing sarcoma in South Korea was 1.01, similar to other Asian countries. While Taiwan study observed a second incidence peak at 45-50 years [17], the occurrence in South Korean population was highest in children and decreased in an age-dependent manner.

During the 19-year period, treatment modalities and survival rates were similar. The 2009-2013 period is peculiar, with approximately $20 \%$ more cases diagnosed during this period. Incidence rates were slightly higher across all age groups; however, the exact cause is elusive. Since the early 2000s, Ewing sarcoma incidence has increased in older patients, presumably related to demographic changes, including an increasingly aging population.

There is a controversy as to whether similar treatment strategies produce similar outcomes as in Euro-American populations for Ewing sarcoma in low-incidence populations. The survival of Korean patients with Ewing sarcoma was lower than that of patients in the United States [18], but was slightly higher than that of Japanese patients [19]. Despite using a different index (overall vs. relative survival), a survival gap of more than $10 \%$ exists between the United States [18] and Korea. The 5-year relative survival rate of patients with Ewing sarcoma in the United States was 77.4\% in children and $65.4 \%$ in AYA [18]. The 5-year OS rates of Korean children and AYA were $64 \%$ and $51 \%$, respectively. Whereas, the survival of Korean patients with Ewing sarcoma appeared to be slightly better than that of Japanese patients [19]. The 5-year survival rates of Japan and South Korea were $49 \%$ and $52 \%$, respectively. Specifically, the survival rates of Japanese children, AYA, and adults were $57.5 \%$, $47.5 \%$, and $24.2 \%$, respectively. Survival data of the elderly patients were not provided. In South Korea, the survival rates of the corresponding age groups were $64 \%, 51 \%, 42 \%$, respectively, and $19 \%$ in the elderly. Leaving ethnicity aside, we observed a
Table 2. Univariate and multivariate analyses of prognostic factors

\begin{tabular}{|c|c|c|}
\hline Variable & Univariate & Multivariate \\
\hline \multicolumn{3}{|l|}{ Sex } \\
\hline Male & Reference & Reference \\
\hline Female & $0.95(0.75-1.20)$ & $1.22(0.78-1.59)$ \\
\hline \multicolumn{3}{|l|}{ Tumor location } \\
\hline Extremity & Reference & Reference \\
\hline Trunk & $1.64(1.26-2.12)$ & $0.95(0.63-1.41)$ \\
\hline Elsewhere & $1.33(0.94-1.90)$ & $1.02(0.64-1.63)$ \\
\hline \multicolumn{3}{|l|}{ Age (yr) } \\
\hline$\leq 14$ & Reference & Reference \\
\hline $15-39$ & $1.54(1.17-2.03)$ & $1.80(1.13-2.84)$ \\
\hline $40-64$ & $1.74(1.20-2.53)$ & $2.91(1.75-4.83)$ \\
\hline$\geq 65$ & $6.35(4.18-9.64)$ & $6.85(3.62-12.98)$ \\
\hline \multicolumn{3}{|c|}{$\begin{array}{l}\text { Presence of metastasis }{ }^{a} \\
\text { (since 2006) }\end{array}$} \\
\hline No & Reference & Reference \\
\hline Yes & $2.92(2.05-4.17)$ & $2.60(1.76-3.84)$ \\
\hline Unknown & $1.19(0.74-1.92)$ & $1.63(1.02-2.61)$ \\
\hline \multicolumn{3}{|l|}{ Treatment $^{\mathrm{b})}$} \\
\hline Local only & Reference & Reference \\
\hline Multidisciplinary & $0.88(0.66-1.16)$ & $1.11(0.75-1.66)$ \\
\hline \multicolumn{3}{|c|}{$\begin{array}{l}\text { a) Data regarding the presence of metastasis was available from } \\
\text { 2006. Accordingly, multivariate analysis was performed using } \\
\text { data from 2006, b)Local only corresponds to surgery and/or } \\
\text { radiotherapy, and multidisciplinary includes surgery, radiother- } \\
\text { apy and chemotherapy. }\end{array}$} \\
\hline
\end{tabular}

difference in treatment practices between the two countries. In our data, $63.2 \%$ of adults and $40.0 \%$ of elderly patients received multidisciplinary treatment, including chemotherapy. In contrast, $38.1 \%$ of adults and $17.8 \%$ of elderly Japanese patients received chemotherapy [19]. Currently available data show that the outcome of Ewing sarcoma in Asian populations is inferior to that of Caucasian populations, suggesting genetic differences [20-22] or lack of clinical studies as possible causes. However, a case series of Korean patients with localized Ewing sarcoma from the Korea Cancer Center Hospital reported a similar outcome to those of Euro-American cases, emphasizing the importance of multidisciplinary treatment [23]. We presume that international studies involving multiple low-incidence populations could be an effective measure to elucidate whether using the similar treatment approach could improve the survival of Ewing sarcoma in a low-incidence population.

The effects of age on the prognosis of Ewing sarcoma are complex. The European study group concluded that age, primary site, and metastasis at diagnosis were poor prognostic factors [24]. According to the U.S. SEER and Japanese Bone 
and Soft Tissue Tumor Registry (BSTTR) data, the survival of children was better than that of AYA and adults $[18,19]$. In contrast, recent studies suggest that older age is not a poor prognostic factor, as adults had similar outcomes to children when treated with chemotherapy $[25,26]$. In a case series of 59 adult patients, in which metastasis at diagnosis was the only predictor of long-term survival [27]. In 76 patients with localized Ewing sarcoma, a poor histologic response to preoperative chemotherapy and tumor location in a centralaxial body region were associated with poorer event-free survival [23]. The KCCR data showed that survival of AYA approximated all patients with Ewing sarcoma. The 5-year survival was poorer after the AYA age and decreased precipitously in the elderly patients ( $42 \%$ in adults, $19 \%$ in the elderly). The California Cancer Registry data did not observe survival differences among adult age groups 20-39, 40-49, $50-59,60-69$, and 70 years $(\mathrm{p}=0.466)$ [28]. The prognostic significance of age in Ewing sarcoma should be validated considering the following factors. First, the biological characteristics of the tumor might be different between children / AYA and adults/elderly. We observed that older patients (adults, elderly) more frequently presented with tumors in the trunk and metastases at the time of diagnosis. Second, the level of treatment could influence the outcome. The accepted standard of care for Ewing sarcoma is multidisciplinary treatment, comprising both local therapy and systemic chemotherapy [1]. Although the majority of patients underwent surgery or radiotherapy, the proportion of patients who received chemotherapy was lower in adults and elderly patients. We presume that poor tolerance to cytotoxic chemotherapy and the presence of comorbidities might have contributed to the less aggressive treatments given to adults and elderly patients.

In conclusion, we described the epidemiology of Ewing sarcoma in South Korea for the first time using the KCCR data. With a similar incidence to other Asian countries, the survival of Korean patients was slightly lower than that of Euro-American cases. We observed that the presence of metastasis and older age at diagnosis were poor prognostic factors. Older patients often presented with axial tumors and metastasis, received less intensive treatment, and had inferior survival rates compared to AYA and children. Future research needs to be focused on improving the outcome of these patients either by actively involving them in clinical trials or by introducing novel agents.

\section{Ethical Statement}

This study was approved by the Institutional Review Board (IRB) of the National Cancer Center (IRB No. NCC 2021-0064), and the requirement for informed consent was waived because it was a secondary analysis of de-identified data.

\author{
Author Contributions \\ Conceived and designed the analysis: Lee JA, Lim J, Won YJ. \\ Collected the data: Won YJ. \\ Contributed data or analysis tools: Lim J, Won YJ. \\ Performed the analysis: Park D. \\ Wrote the paper: Lee JA, Lim J, Won YJ. \\ Interpretation and editing: Jin HY, Park M. \\ Review and Comment: Park HJ, Park JW, Kim JH, Kang HG.
}

\section{Conflicts of Interest}

Conflict of interest relevant to this article was not reported.

\section{Acknowledgments}

This work was supported by a research grant (No. 1910132-3) from the National Cancer Center, Republic of Korea.

\section{References}

1. Riggi N, Suva ML, Stamenkovic I. Ewing's sarcoma. N Engl J Med. 2021;384:154-64.

2. Jawad MU, Cheung MC, Min ES, Schneiderbauer MM, Koniaris LG, Scully SP. Ewing sarcoma demonstrates racial disparities in incidence-related and sex-related differences in outcome: an analysis of 1631 cases from the SEER database, 1973-2005. Cancer. 2009;115:3526-36.

3. Worch J, Matthay KK, Neuhaus J, Goldsby R, DuBois SG. Ethnic and racial differences in patients with Ewing sarcoma. Cancer. 2010;116:983-8.

4. Curado MP, Edwards B, Shin HR, Storm H, Ferlay J, Heanue $\mathrm{M}$, et al. Cancer incidence in five continents. Lyon: IARC Press; 2007.

5. Anfinsen KP, Devesa SS, Bray F, Troisi R, Jonasdottir TJ, Bru- land OS, et al. Age-period-cohort analysis of primary bone cancer incidence rates in the United States (1976-2005). Cancer Epidemiol Biomarkers Prev. 2011;20:1770-7.

6. Whelan J, McTiernan A, Cooper N, Wong YK, Francis M, Vernon $S$, et al. Incidence and survival of malignant bone sarcomas in England 1979-2007. Int J Cancer. 2012;131:E508-17.

7. Juergens C, Weston C, Lewis I, Whelan J, Paulussen M, Oberlin $\mathrm{O}$, et al. Safety assessment of intensive induction with vincristine, ifosfamide, doxorubicin, and etoposide (VIDE) in the treatment of Ewing tumors in the EURO-E.W.I.N.G. 99 clinical trial. Pediatr Blood Cancer. 2006;47:22-9.

8. Miser JS, Krailo MD, Tarbell NJ, Link MP, Fryer CJ, Pritchard DJ, et al. Treatment of metastatic Ewing's sarcoma or primitive neuroectodermal tumor of bone: evaluation of combina- 
tion ifosfamide and etoposide: a Children's Cancer Group and Pediatric Oncology Group study. J Clin Oncol. 2004;22:2873-6.

9. Perlman EJ, Dickman PS, Askin FB, Grier HE, Miser JS, Link MP. Ewing's sarcoma: routine diagnostic utilization of MIC2 analysis: a Pediatric Oncology Group/Children's Cancer Group Intergroup Study. Hum Pathol. 1994;25:304-7.

10. Zucker JM, Henry-Amar M. Therapeutic controlled trial in Ewing's sarcoma. Report on the results of a trial by the Clinical Cooperative Group on Radio- and Chemotherapy of the E.O.R.T.C. Eur J Cancer. 1977;13:1019-23.

11. Grier HE, Krailo MD, Tarbell NJ, Link MP, Fryer CJ, Pritchard DJ, et al. Addition of ifosfamide and etoposide to standard chemotherapy for Ewing's sarcoma and primitive neuroectodermal tumor of bone. N Engl J Med. 2003;348:694-701.

12. Korean Statistical Information Service [Internet]. Daejeon: Statisticak Korea; 2021 [cited 2021 Feb 1]. Available from: https://kosis.kr/eng/statisticsList/statisticsListIndex.do? menuId=M_01_01\&vwcd=MT_ETITLE\&parmTabId=M_ 01_01.

13. Segi M. Cancer mortality for selected sites in 24 countries (1950-57). Sendai: Tohoku University School of Medicine; 1960.

14. Young JL, Roffers SD, Ries LA, Fritz AG, Hurlbut AA. SEER summary staging manual, 2000: coded and coding instructions. Bethesda, MD: National Cancer Institute; 2001.

15. Esteve J, Benhamou E, Raymond L. Statistical methods in cancer research. Vol. IV. Descriptive epidemiology. Oxford: Oxford University Press; 1994.

16. Cox DR. Regression models and life tables. J R Stat Soc Series B. 1972;34:187-220.

17. Hung GY, Horng JL, Yen HJ, Yen CC, Chen WM, Chen PC, et al. Incidence patterns of primary bone cancer in taiwan (2003-2010): a population-based study. Ann Surg Oncol. 2014;21:2490-8.

18. Keegan TH, Ries LA, Barr RD, Geiger AM, Dahlke DV, Pollock $\mathrm{BH}$, et al. Comparison of cancer survival trends in the United States of adolescents and young adults with those in children and older adults. Cancer. 2016;122:1009-16.

19. Fukushima T, Ogura K, Akiyama T, Takeshita K, Kawai A. Descriptive epidemiology and outcomes of bone sarcomas in adolescent and young adult patients in Japan. BMC Musculoskelet Disord. 2018;19:297.

20. Arvand A, Denny CT. Biology of EWS/ETS fusions in Ewing's family tumors. Oncogene. 2001;20:5747-54.

21. Burchill SA. Ewing's sarcoma: diagnostic, prognostic, and therapeutic implications of molecular abnormalities. J Clin Pathol. 2003;56:96-102.

22. Kojima T, Asami S, Chin M, Yoshida Y, Mugishima H, Suzuki T. Detection of chimeric genes in Ewing's sarcoma and its clinical applications. Biol Pharm Bull. 2002;25:991-4.

23. Lee JA, Kim DH, Cho J, Lim JS, Koh JS, Yoo JY, et al. Treatment outcome of Korean patients with localized Ewing sarcoma family of tumors: a single institution experience. Jpn J Clin Oncol. 2011;41:776-82.

24. Cotterill SJ, Ahrens S, Paulussen M, Jurgens HF, Voute PA, Gadner $\mathrm{H}$, et al. Prognostic factors in Ewing's tumor of bone: analysis of 975 patients from the European Intergroup Cooperative Ewing's Sarcoma Study Group. J Clin Oncol. 2000;18:3108-14.

25. Fizazi K, Dohollou N, Blay JY, Guerin S, Le Cesne A, Andre F, et al. Ewing's family of tumors in adults: multivariate analysis of survival and long-term results of multimodality therapy in 182 patients. J Clin Oncol. 1998;16:3736-43.

26. Bacci G, Ferrari S, Comandone A, Zanone A, Ruggieri P, Longhi A, et al. Neoadjuvant chemotherapy for Ewing's sarcoma of bone in patients older than thirty-nine years. Acta Oncol. 2000;39:111-6.

27. Martin RC 2nd, Brennan MF. Adult soft tissue Ewing sarcoma or primitive neuroectodermal tumors: predictors of survival? Arch Surg. 2003;138:281-5.

28. Lee J, Hoang BH, Ziogas A, Zell JA. Analysis of prognostic factors in Ewing sarcoma using a population-based cancer registry. Cancer. 2010;116:1964-73. 